\title{
Helly-Type Theorems for Spheres
}

\author{
Hiroshi Maehara
}

Ryukyu University, Nishihara, Okinawa, Japan

Dedicated to Professor Itiro Tamura on his 60 th birthday

\begin{abstract}
We prove that (i) a family $F$ of at least $n+3$ spheres in $E^{n}$ has nonempty intersection if each $n+1$ spheres of $\mathbf{F}$ have nonempty intersection, and (ii) if a family $\mathbf{F}$ of spheres in $E^{\prime \prime}$ has nonempty intersection, then there exist $n+1$ or fewer spheres in $\mathbf{F}$ whose intersection coincides with the intersection of all spheres of $\mathbf{F}$.
\end{abstract}

\section{Introduction}

Helly's theorem is one of the most important results in the study of combinatorial geometry of convex sets in Euclidean space $E^{n}$.

Theorem (Helly [2]). Let $\mathbf{F}$ be a finite family of at least $n+1$ convex sets in $E^{n}$. If every $n+1$ members of $\mathrm{F}$ have a point in common, then there is a point common to all members of $\mathbf{F}$.

Here we present similar results for families of spheres in $E^{n}$. Let us say that a family $\mathbf{F}$ of sets has the Helly-n-property if the intersection of $\mathbf{F}$ is nonempty or there are $n+1$ or fewer members of $\mathbf{F}$ which have empty intersection. Then Helly's theorem states that every finite family of convex sets in $E^{n}$ has the Helly-n-property. We will show that a family $\mathbf{F}$ of spheres in $E^{n}$ whose centers are all lying on an $m$-dimensional flat has the Helly- $(m+1)$-property (Theorem 1 ), and if $\mathbf{F}$ has nonempty intersection then there exist $m+1$ or fewer spheres in F whose intersection coincides with the intersection of all spheres in $\mathbf{F}$ (Theorem 2). Further, if a family of spheres in $E^{n}$ contains at least $n+3$ distinct spheres then the family has the Helly-n-property (Theorem 3 ). 


\section{Spheres with Centers on a Flat}

A sphere $S$ of radius $r$ in Euclidean $n$-space $E^{n}$ consists of all points at distance $r$ from some fixed point called the center of $S$. If $r=1$ then $S$ is called a unit sphere. We admit the case of $r=0$ (then $S$ consists of a single point). The intersection $S \cap L$ of a sphere $S$ and a flat (affine subspace) $L$ in $E^{n}$ is called a sphere in $L$, provided that $S \cap L$ is nonempty. The intersection of all sets in a family $\mathbf{F}$ is denoted by $\bigcap \mathbf{F}$.

Theorem 1. Let $\mathbf{F}$ be a family of spheres in $E^{n}$ with centers on a flat $L$ of dimension $m$. Suppose that every subfamily consisting of at most $m+2$ spheres in $\mathbf{F}$ has nonempty intersection. Then $\mathrm{F}$ has nonempty intersection.

Remark. the number $m+2$ cannot be reduced, see Fig. 1 in which $m=1, n=2$, and each two circles have two common points, but all circles have no point in common.

Proof. Since a family of compact sets with the finite intersection property has nonempty intersection, and since every sphere is compact, it is enough to prove the theorem when $\mathbf{F}$ is finite. So, we may assume that $\mathbf{F}$ is finite. First we consider the case $m=1$. Suppose $y \in S_{1} \cap S_{j}\left(S_{i}, S_{,} \in \mathbf{F}\right)$, and let $H_{y}$ be the hyperplane passing through $y$ and perpendicular to $L$ at $z$. Then $S_{i} \cap S_{\text {, is }}$ the sphere in the hyperplane $H_{y}$ with center $z$ and radius $y z$. Hence $S_{1} \cap S_{2} \cap S_{3} \neq \varnothing\left(S_{1} \in \mathbf{F}\right.$, $i=1,2,3)$ implies that $S_{1} \cap S_{2}=S_{1} \cap S_{2} \cap S_{3}$. Therefore every sphere of $F$ contains, say, $S_{1} \cap S_{2}$, and hence $\bigcap \mathbf{F} \neq \varnothing$. Now the general case. We proceed by induction on $n$. If $n=1$ then $m=1$ and the theorem is true. Let $n>1$ and suppose that the theorem is true for dimension $<n$. We may assume that there is a sphere $S_{0}$ of positive radius in $\mathbf{F}$ (otherwise, the theorem is trivial). Let $\mathbf{G}=$ $\left\{S_{0} \cap S: S \in \mathbf{F}, S \neq S_{0}\right\}$. Then every subfamily of $\mathbf{G}$ of size at most $m+1$ has nonempty intersection, and we need only show that $\bigcap \mathbf{G}$ is nonempty. Since $\mathbf{F}$ is finite, and assuming $m \geq 2$, we can take a point $x$ on $S_{0} \cap L$ which does not lie on any other sphere in F. Let $H$ be the hyperplane tangent to $S_{0}$ at the point antipodal to $x$. By the stereographic projection $f$ of $S_{0}$ from $x$ to $H$, each member $T$ of $\mathbf{G}$ is carried onto a sphere in $H$. The centers of the spheres of

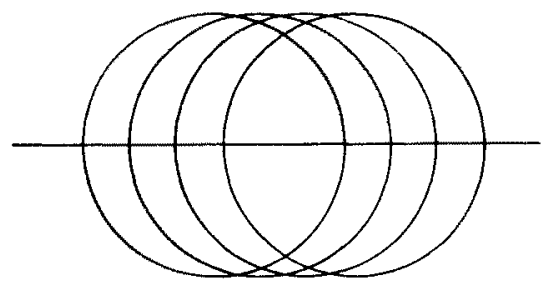

Fig. 1 




Fig. 2

$f(\mathbf{G}):=\{f(T): T \in \mathbf{G}\}$ lie on the $(m-1)$-dimensional flat $H \cap L$, and every subfamily of $f(\mathbf{G})$ of size at most $m+1$ has nonempty intersection. Since $H$ is $(n-1)$-dimensional, $\cap f(\mathbf{G})$ must be nonempty by the inductive hypothesis. Hence $\cap \mathbf{G}$ is nonempty.

Corollary 1. Let $\mathbf{F}$ be a family of at least $n+2$ spheres in $E^{n}$. If every $n+2$ spheres in $\mathbf{F}$ have a point in common, then $\mathbf{F}$ has nonempty intersection.

Theorem 2. Let $\mathbf{F}$ be a family of spheres in $E^{n}$ with nonempty intersection. Suppose that the centers of the spheres in $\mathbf{F}$ all lie on a flat of dimension $m$. Then there exist $m+1$ or fewer spheres in $\mathbf{F}$ whose intersection is equal to the intersection $\bigcap \mathbf{F}$.

Remark. The number $m+1$ cannot be reduced, see Fig. 2 in which $m=n=2$ and any two circles have two points in common, but $\bigcap \mathbf{F}$ consists of one point. It is also impossible to drop the assumption $\mathbf{F} \neq \varnothing$, see Fig. 3 .

Proof. By induction on $n$. If $n=1$ then the theorem is obvious. Suppose that the theorem is true for $n-1(n>1)$, and let us consider the case of dimension $n$. Since the case $m=1$ is clear, we may further suppose $m \geq 2$. First we show that there is a finite subfamily $\mathbf{K}_{0}$ of $\mathbf{F}$ such that $\bigcap \mathbf{K}_{0}=\bigcap \mathbf{F}$. To see this let $\mathbf{K}_{0}$ be a finite subfamily of $\mathbf{F}$ which attains the minimum value of $\operatorname{dim}(\cap \mathbf{K})$ among all finite-subfamilies $\mathbf{K}$ of $\mathbf{F}$. (Here $\operatorname{dim}(X)$ means the dimension of the flat spanned by $X, \operatorname{dim}($ one point $)=0$, and $\operatorname{dim}(\varnothing)=-1$.) Then clearly $\bigcap \mathbf{K}_{0}$ is a

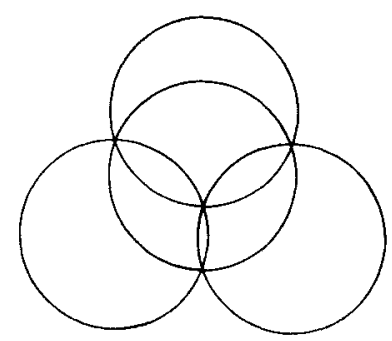

Fig. 3 
sphere in some flat. Suppose $\bigcap \mathbf{K}_{0} \neq \bigcap \mathbf{F}$. Then there must be a sphere $S$ in $\mathbf{F} \backslash \mathbf{K}_{0}$ such that $\operatorname{dim}\left(\cap \mathbf{K}_{0} \cap S\right)<\operatorname{dim}\left(\cap \mathbf{K}_{0}\right)$, which contradicts the choice of $\mathbf{K}_{0}$. Thus $\bigcap \mathbf{K}_{0}=\bigcap \mathbf{F}$. Now, we may assume that there is a sphere $S_{0}$ of radius $>0$ in $\mathbf{K}_{0}$. Let $\mathbf{G}=\left\{S_{0} \cap S: S \in \mathbf{K}_{0}, S \neq S_{0}\right\}$. Then $\bigcap \mathbf{F}=\bigcap \mathbf{G}$. Since $\mathbf{K}_{0}$ is finite and $m \geq 2$, there is a point $x$ on $S_{0} \cap L$ which does not lie on any other sphere in $\mathbf{K}_{0}$. Let $f$ be the stereographic projection of $S_{0}$ from $x$ to the hyperplane $H$ tangent to $S_{0}$ at the point antipodal to $x$. Then $f(\mathbf{G}):=\{f(T): T \in \mathbf{G}\}$ has nonempty intersection, and the centers of the spheres in $f(\mathbf{G})$ all lie on the $(m-1)$-dimensional flat $H \cap L$. Since $f(\mathbf{G})$ is a family of spheres in $(n-1)$-dimensional flat $H$ and $f(\mathbf{G})$ has nonempty intersection, it follows from the inductive hypothesis that there are spheres $S_{1}, \ldots, S_{k}(k \leq m)$ in $\mathbf{K}_{0}$ such that the intersection of $f\left(S_{0} \cap S_{l}\right)$, $i=1, \ldots, k$, is equal to $\bigcap f(\mathbf{G})$. Therefore the intersection of $S_{i}, i=0,1, \ldots, k$, is equal to $\bigcap \mathbf{F}$.

Corollary 2. Let $\mathbf{F}$ be a family of spheres in $E^{n}$ with nonempty intersection. Then there exist $n+1$ or fewer spheres in $E^{n}$ whose intersection coincides with the intersection of all spheres in $\mathbf{F}$.

\section{A Family of at Least $n+3$ Spheres}

Theorem 3. Let $\mathbf{F}$ be a family of at least $n+3$ different spheres in $E^{n}$. If any $n+1$ spheres in $\mathbf{F}$ have a common point, then all spheres in $\mathbf{F}$ have a common point.

Remark. The number $n+1$ cannot be replaced by $n$, see Proposition 1 below. It is also impossible to reduce the number $n+3$ to $n+2$, see Fig. 3 .

First we prove the following lemma.

Lemma. Let $\mathbf{G}=\left\{S_{i}: i=1, \ldots, n+2\right\}$ be a family of $n+2$ different spheres in $E^{n}$ such that

$$
\bigcap\left(\mathbf{G} \backslash\left\{S_{i}\right\}\right) \ni x_{i}, \quad i=1, \ldots, n+2, \quad \text { and } \bigcap \mathbf{G}=\varnothing
$$

Let $X=\left\{x_{i}: i=1, \ldots, n+2\right\}$ and $Z=\left\{z_{i}: i=1, \ldots, n+2\right\}$, where $z_{1}$ is the center of $S_{i}$. Then for each $i$, both of $Z \backslash\left\{z_{i}\right\}$ and $X \backslash\left\{x_{i}\right\}$ span $E^{n}$. Furthermore, for $1 \leq i \neq j \leq$ $n+2$,

$$
\bigcap\left(\mathbf{G} \backslash\left\{S_{i}\right\}\right)=\left\{x_{i}\right\} \text { and } \cap\left(\mathbf{G} \backslash\left\{S_{i}, S_{i}\right\}\right)=\left\{x_{i}, x_{j}\right\}
$$

Proof. The case $n=1$ is obvious, so we assume $n \geq 2$. Suppose that $Z \backslash\left\{z_{1}\right\}$ is contained in a flat of dimension $m<n$. Then, by Theorem 2 , there are $m+1(\leq n)$ spheres in $\mathbf{G} \backslash\left\{S_{i}\right\}$ whose intersection coincides with $\bigcap\left(\mathbf{G} \backslash\left\{S_{i}\right\}\right)$. These $m+1$ spheres and the sphere $S_{i}$ have a common point because any $n+1$ spheres of $G$ have a common point. Hence $\bigcap\left(\mathbf{G} \backslash\left\{S_{i}\right\}\right)$ and $S_{i}$ have a common point, that is, $\cap \mathbf{G} \neq \varnothing$, a contradiction. Therefore $Z \backslash\left\{z_{i}\right\}$ must span $E^{n}$. Suppose now $x_{i}$ lies 
on the flat spanned by $X \backslash\left\{x_{i}, x_{j}\right\}, i \neq j$. then, since $X \backslash\left\{x_{i}\right\}$ is spherical (i.e., lying on the sphere $S_{1}$ ), every sphere passing through all the points of $X \backslash\left\{x_{1}, x_{1}\right\}$ also passes through $x_{i}$. Hence $S_{\text {, }}$ (which passes through all points of $X \backslash\left\{x_{1}\right\}$ ) must pass through $x_{i}$. This means $\cap \mathbf{G} \ni x_{i}$, a contradiction. Hence $X \backslash\left\{x_{i}\right\}$ spans $E^{n}$. Finally, to show $\bigcap\left(\mathbf{G} \backslash\left\{S_{i}\right\}\right)=\left\{x_{i}\right\}$, suppose that $\bigcap\left(\mathbf{G} \backslash\left\{x_{i}\right\}\right)$ contains another point $y_{i} \neq x_{i}$. Then the set $Z \backslash\left\{z_{i}\right\}$ must lie on the hyperplane perpendicularly bisecting the line segment $x_{i} y_{i}$, which is a contradiction. Thus $\cap\left(\mathbf{G} \backslash\left\{X_{t}\right\}\right)=\left\{x_{i}\right\}$. Similarly, since $Z \backslash\left\{z_{1}, z_{j}\right\}$ spans a hyperplane, it follows that $\cap\left(\mathbf{G} \backslash\left\{S_{i}, S,\right\}\right)$ consists of at most two points. And since

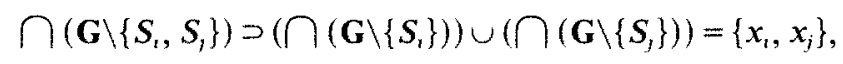

it follows that $\cap\left(\mathbf{G} \backslash\left\{S_{1}, S_{y}\right\}\right)=\left\{x_{i}, x_{j}\right\}$.

Proof of Theorem 3. We will show that all $n+2$ spheres in $\mathbf{F}$ have a point in common. Then, by Corollary 1 , all spheres in $\mathbf{F}$ have a point in common. Suppose that there is a subfamily $\mathbf{G}=\left\{S_{i}: i=1, \ldots, n+2\right\}$ of $n+2$ spheres of $\boldsymbol{F}$ with empty intersection. Let $X=\left\{x_{i}: i=1, \ldots, n+2\right\}$ and $Z=\left\{z_{i}: i=1, \ldots, n+2\right\}$ be as in

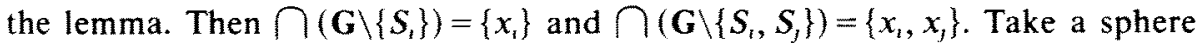
$S_{n+3}$ from $\mathbf{F} \backslash \mathbf{G}$. Then

$$
\varnothing \neq \bigcap\left(\mathbf{G} \backslash\left\{S_{1}, S_{j}\right\}\right) \cap S_{n+3}=\left\{x_{i}, x_{j}\right\} \cap S_{n+3}, \quad 1 \leq i \neq j \leq n+2 .
$$

Hence $S_{n+3}$ contains at least $n+1$ points of $X$. Without loss of generality, we may assume that $X \backslash\left\{x_{1}\right\} \subset S_{n+3}$. Then, since $X \backslash\left\{x_{1}\right\} \subset S_{1}$ and $X \backslash\left\{x_{1}\right\}$ spans $E^{n}$, $S_{n+3}$ must be identical with $S_{1}$, which is a contradiction.

Combining Corollary 2 with Theorem 3 , we have the following.

Corollary 3. Let $\mathbf{F}$ be a family of at least $n+3$ spheres in $E^{n}$. Then there exist $n+1$ or fewer spheres in $\mathbf{F}$ whose intersection coincides with the intersection of all spheres in $\mathbf{F}$.

Now we show that the number $n+1$ in Theorem 3 cannot be replaced by $n$.

Proposition 1. For any $N>n$, there exists a family $\mathbf{F}$ of $N$ different unit spheres in $E^{n}$ such that all $n$ spheres in $\mathbf{F}$ have a common point, but no $n+1$ spheres in $\mathbf{F}$ have a common point.

Proof. Choose $N$ points on a sphere of radius $\frac{1}{2}$ in $E^{n}$ in such a way that no $n+1$ points of them lie on a hyperplane (that is, these $N$ points are in general position). This is clearly possible because no finite number of hyperplanes can cover a sphere of positive radius. Let $\mathbf{F}$ be the family of the $N$ unit spheres centered at these $N$ points. Then any $n$ spheres of $F$ have exactly two points in common, but no $n+1$ spheres in $F$ have a point in common. 


\section{A Family of Unit Spheres}

In Theorem 3 we cannot reduce the size of $\mathbf{F}$ to $n+2$, since for every $n$ there exists a family $\mathbf{G}$ of $n+2$ spheres in $E^{n}$ such that any $n+1$ spheres of $\mathbf{G}$ have a common point, but $\cap \mathbf{G}=\varnothing$. The plane case $(n=2)$ is depicted in Fig. 3 . This plane case is of special interest: if some three circles of $\mathbf{G}$ are of unit radius, then the rest is also a unit circle. (Proof is not difficult.) Thus, even if we restrict ourselves to unit spheres, it is generally impossible to reduce the least size $n+3$ of $\mathbf{F}$ in Theorem 3 to $n+2$. But how about the case $n \neq 2$ ? Is there a family $\mathbf{G}$ of $n+2$ unit spheres in $E^{n}(n \neq 2)$ such that any $n+1$ spheres of $G$ have a common point and $\cap \mathbf{G}=\varnothing$ ? We consider this in some detail.

Let $\Delta$ be an $n$-simplex in $E^{n}$. By the $c$-center and the $c$-radius (denoted by $R(\Delta)$ ) of $\Delta$, we mean the center and the radius of the circumscribed sphere of $\Delta$. Let $w$ be the $c$-center of $\Delta$ and let $y_{i}, i=1, \ldots, n+1$, be the feet of the perpendicular from $w$ to the $n+1$ hyperplanes determined by the $n+1$ faces of $\Delta$. Let us denote by $\Delta^{\prime}$ the (possibly degenerate) simplex spanned by $y_{1}, i=$ $1, \ldots, n+1$. If $\Delta^{\prime}$ is degenerate then we set its $c$-radius $R\left(\Delta^{\prime}\right)=\infty$. A simplex is said to be critical if its $c$-center lies on the hyperplane determined by one of its faces, otherwise the simplex is said to be general.

Proposition 2. If $\Delta$ is a critical simplex then $R\left(\Delta^{\prime}\right) / R(\Delta)=\frac{1}{2}$.

Proof. Suppose that $\Delta$ is a critical $n$-simplex with vertices $z_{1}, i=1, \ldots, n+1$, in $E^{n}$ whose $c$-center $w$ lies on the hyperplane determined by the face opposite $z_{n+1}$. Then $w$ is a vertex of $\Delta^{\prime}$. Let $y_{i}, i=1, \ldots, n$, be the other vertices of $\Delta^{\prime}$. Then, since the angle $\left\langle w y_{i} z_{n+1}=90^{\circ}\right.$ for $i=1, \ldots, n$, the $n+1$ points $w, y_{1}, \ldots, y_{n}$ lie on the sphere with diameter $w z_{n+1}$. Hence $2 R\left(\Delta^{\prime}\right)=w z_{n+1}=R(\Delta)$.

Proposition 3. The following two statements are equivalent:

(1) There exists a family $\mathbf{G}=\left\{S_{i}: i=1, \ldots, n+2\right\}$ of unit spheres in $E^{n}$ such that $\cap\left(\mathbf{G} \backslash\left\{S_{i}\right\}\right) \neq \varnothing$ for each $i$, but $\cap \boldsymbol{G}=\varnothing$.

(2) There exists a general n-simplex $\Delta$ in $E^{n}$ such that $R(\Delta)=2 R\left(\Delta^{\prime}\right)$.

Proof. (1) $\rightarrow(2)$. Let $X$ and $Z$ be as in the lemma, and let $\Delta$ be the simplex spanned by $z_{1}, \ldots, z_{n+1}$. Then $x_{n+2}$ is the $c$-center of $\Delta$, and since


perpendicularly bisects the line segment $x_{i} x_{n+2}$ at, say, $y_{1}$. Since $x_{n+2} \neq y_{i}, i<n+2$, $\Delta$ is a general simplex. Further, the simplex $\Delta^{\prime \prime}$ spanned by $x_{1}, \ldots, x_{n+1}$ has unit $c$-radius (because all vertices lie on $S_{n+2}$ ), and $\Delta^{\prime \prime}$ is similar to the simplex $\Delta^{\prime}$ spanned by $y_{1}$ 's with center of similitude $x_{n+2}$ and ratio 2 . Hence $R\left(\Delta^{\prime}\right)=\frac{1}{2}$.

(2) $\rightarrow(1)$. Suppose there exists a general $n$-simplex $\Delta$ in $E^{n}$ of unit $c$-radius such that $R\left(\Delta^{\prime}\right)=\frac{1}{2}$. Let $z_{i}, i=1, \ldots, n+1$, be the vertices of $\Delta$ and $x_{n+2}$ be the $c$-center of $\Delta$. For each $i<n+2$, let $x_{i}$ be the reflection of $x_{n+2}$ with respect to the hyperplane determined by the face of $\Delta$ opposite the vertix $z_{1}$. Then, since $\Delta$ is general, the points $x_{i}, i=1, \ldots, n+1$, are all different, and the simplex $\Delta^{\prime \prime}$ spanned by these points is similar to $\Delta^{\prime}$. So $R\left(\Delta^{\prime \prime}\right)=1$. Let $z_{n+2}$ be the $c$-center 
of $\Delta^{\prime \prime}$. Let $\mathrm{G}$ be the family of unit spheres $S_{i}$ centered at $z_{\imath}, i=1, \ldots, n+2$. Then it is easy to check that

$$
\bigcap\left(\mathbf{G} \backslash\left\{S_{1}\right\}\right)=\left\{x_{i}\right\} \quad \text { for } i=1, \ldots, n+2, \text { but } \cap \mathbf{G}=\varnothing .
$$

In the plane case $n=2, R\left(\Delta^{\prime}\right) / R(\Delta)=\frac{1}{2}$ holds for any triangle $\Delta$. However this is not the case in higher dimensions. It is not difficult to see that $R\left(\Delta^{\prime}\right) / R(\Delta)=$ $1 / n$ for a regular $n$-simplex $\Delta$ of unit $c$-radius in $E^{n}$. In the case $n>2$, if we move a vertex $z_{1}$ of a regular $n$-simplex $\Delta$ along the perpendicular to the opposite face of $z_{1}$ (keeping the other vertices fixed), then $R\left(\Delta^{\prime}\right) / R(\Delta)$ varies from $1 / n$ to $\infty$. And $R\left(\Delta^{\prime}\right) / R(\Delta)$ takes the value $\frac{1}{2}$ only when $\Delta$ becomes critical (this is also not difficult to check). This suggests the following conjecture.

Conjecture. Let $\mathbf{F}$ be a family of at least $n+2$ unit spheres in $E^{n}$. If $n \neq 2$ and any $n+1$ spheres in $\mathbf{F}$ have a common point, then all spheres in $\mathbf{F}$ have a common point.

\section{Acknowledgment}

1 would like to thank the referee for helpful advices.

\section{References}

1. L. Danzer, B. Grünbaum, and V. Klee, Helly's theorem and its relatives, Proceedings of Symposia in Pure Mathematics, Vol. 7, American Mathematical Society, Providence, RI, 1963.

2. E. Helly, Über Mengen konvexer Körper mit gemeinschaftlichen Punkten, Jahresber. Deuisch. Math.-Verein. 32 (1923), 175-176.

Received September 8, 1986, and in revised form December 17, 1986. 